Project 90138

\title{
Portable Analyzer Based on Microfluidics/Nanoengineered Electrochemical Sensors for in Situ Characterization of Mixed Wastes
}

\author{
Wang, Joseph \\ New Mexico State University
}

RESULTS TO DATE: PROGRESS REPORT Award: DE-FG02-04ER63732 Title: Portable Analyzer Based on Microfluidic/Nanoengineered Electrochemical Sensors for in Situ Characterization of Mixed Wastes PI: Dr. Joseph Wang (In Collaboration with the PNNL PI Dr. Y. Lin). Objective of Research: This research effort aims at developing a portable analytical system for fast, sensitive, and inexpensive, on-site monitoring of toxic transition metals and radionuclides in contaminated DOE Sites. The portable devices will be based on Microscale Total Analytical systems ( -TAS) or 'Lab-on-a-chip' in combination with electrochemical (stripping-voltammetric) sensors. The resulting microfluidics/electrochemical sensor system would allow testing for toxic metals to be performed more rapidly, inexpensively, and reliably in a field setting. Progress Summary/Accomplishments: This report summarizes the ASU activity over the second year of the project. In accordance to our original objectives our studies have focused on various fundamental and practical aspects of sensing and microchip devices for monitoring metal contaminants. As described in this section, we have made a substantial progress, and introduced effective routes for improving the on-site detection of toxic metals and for interfacing microchips with the real world. This activity has already resulted in 7 research papers (published or in press in major international journals). The electrochemical sensors being developed rely on the highly sensitive adsorptive stripping voltammetry (AdSV) technique to detect metal ions of interest to the DOE, particularly uranium and chromium. Traditionally, AdSV measurements of $\mathrm{U}$ and $\mathrm{Cr}$ require the use of mercury electrodes which are not suitable attractive for field deployment. Our initial goal was thus to replace these toxic mercury electrodes with 'environmentally-friendly' sensor materials. In particular, we demonstrated recently that bismuth-film electrodes offer high-quality measurements of heavy metals that compare favorably with that of mercury electrodes. Bismuth is a 'green' element, with very low toxicity, and widespread pharmaceutical use. A major effort of our activity this year has been devoted to the development of a 'mercury-free' uranium sensor based on the bismuth film electrode. Bismuth-coated carbon-fiber electrodes have thus been successfully applied for adsorptive-stripping voltammetric measurements of trace uranium in the presence of the cupferron complexing agent. The new protocol is based on the accumulation of the uraniumcupferron complex at a preplated bismuth film electrode held at $-0.30 \mathrm{~V}$ (vs. $\mathrm{Ag} / \mathrm{AgCl}$ ), followed by a negatively-sweeping square-wave voltammetric waveform. Factors influencing the stripping performance, including the film preparation, solution $\mathrm{pH}$, cupferron concentration, adsorption potential and time have been optimized (Figure 1). The resulting performance compares well with that observed for analogous measurements at mercury film electrodes. The response is linear up to $50 \mathrm{~g} / \mathrm{L}$, with a detection limit of 0.3 $\mathrm{g} / \mathrm{L}$ (Figure 2). Good reproducibility was indicated from a series of 15 repetitive measurements of $50 \mathrm{~g} / \mathrm{L}$ uranium that resulted in reproducible uranium peaks, with a relative standard deviation of $3.8 \%$. Potential interferences are examined. Applicability to sea water samples is demonstrated. The attractive behavior of the new "mercury-free" uranium sensor holds great promise for on-site environmental and industrial monitoring of uranium. Similarly, we developed bismuth-based 'mercury-free' sensors for trace vanadium and molybdenum. The new devices are based on the preconcentration of the molybdenum- or vanadium chloranilic-acid complexes at a preplated bismuth film electrode held at $-0.55 \mathrm{~V}$ (vs. Ag/AgCl), followed by a negatively-sweeping square-wave voltammetric scan. Factors influencing the adsorptive stripping performance, including different ligands, solution $\mathrm{pH}$, CAA concentration, precoencentration time and potential, have been optimized. The response compares favorably with that observed at mercury film electrodes, and is linear over the 5 - $50 \mathrm{~g} / \mathrm{L}$ Mo concentration range (one min preconcentration; Figure 3). A detection limit of $0.2 \mathrm{~g} / \mathrm{L}$ molybdenum is obtained following a $10 \mathrm{~min}$ accumulation. High stability is indicated from the reproducible response of a $100 \mathrm{~g} / \mathrm{L}$ molybdenum solution $(\mathrm{n}=60 ; \mathrm{RSD}=2.6 \%$ ). Applicability to groundwater and seawater samples was demonstrated. Our microchip activity involved the 
development of a novel and effective 'world-to-chip' interface. For this purpose, we coupling of hydrodynamically flowing stream to a microchip capillary electrophoresis (CE) for continuous assays of liquid samples. The new interface relies on mounting the sample tubing onto a sharp inlet tip and allows rapid, convenient and reproducible electrokinetic loading from a continuously flowing stream directly into the narrow separation microchannel (Figure 4). The sharp inlet interface is characterized by its efficiency, stability and simplicity. The effect of the sample flow rate, applied voltages and other relevant variables, is described. It was found that the peak intensity is independent of the flow rate. The performance of the new interface is illustrated for on-line CE-electrochemical monitoring of phenolic and explosive compounds. Conditions simulating continuous long-term monitoring, led to a highly stable response for a 15 ppm 1,3,5trinitrobenzene solution ( $\mathrm{RSD}=3.7 \%, \mathrm{n}=40$ ). Such ability to continuously introduce flowing samples into micrometer channels makes "Lab-on-a-chip" devices highly compatible with real-life monitoring applications.

DELIVERABLES: Publications (from the ASU team of Prof. Wang): 1. "Adsorptive Stripping Voltammetric Measurements of trace Uranium at Bismuth Film Electrodes", L. Lin, J. Wang, Y. Lin and W. Sadik, Anal. Chim. Acta 535(2005)9. 2. "Adsorptive Stripping Voltammetric Measurements of Trace Molybdenum at the Bismuth Film Electrode", J. Wang, S. Thongngamdee and D. Lu, Electroanalysis, in press. 3. "Catalytic Adsorptive Stripping Determination of Trace Chromium (VI) at Bismuth Film Electrodes", L. Lin, N. Lawrence, S. Thongngamdee, J. Wang and Y. Lin, Talanta, 65(2005)144. 4. "Stripping analysis at Bismuth Electrodes: A Review", J. Wang, Electroanalysis, 17(2005)1341. 5. "Catalytic Adsorptive Stripping Voltammetric Measurements of Trace Vanadium at Bismuth Film Electrodes", J. Wang, D. Lai, S. Thongngamdee, Y. Lin and W. Sadik, submitted. 6. "Electrochemical Sensors for Environmental Monitoring: Design, Development and Applications", Deepa G. Patil, G. Hanrahan, and J. Wang, J. Environ. Monit., 6(2004)657. 7. "Continuous Monitoring with Microfabricated Capillary Electrophoresis Chip Devices", J. Wang, W. Siangproh, S. Thongngamdee and O. Chailapakul, Analyst, 130(2005)1390 